\title{
On the dynamics of the Meissner effect
}

\author{
J. E. Hirsch \\ Department of Physics, University of California, San Diego, La Jolla, CA 92093-0319
}

\begin{abstract}
The question of how a metal becoming superconducting expels a magnetic field is addressed. It is argued that the conventional theory of superconductivity has not answered this question despite its obvious importance. We argue that the growth of the superconducting into the normal region and associated expulsion of magnetic field from the superconducting region can only be understood if it is accompanied by motion of charge from the superconducting into the normal region. From a microscopic point of view it is shown that the perfect diamagnetism of superconductors requires that superconducting electrons reside in orbits of spatial extent $2 \lambda_{L}$, with $\lambda_{L}$ the London penetration depth. Associated with this physics, the spin-orbit interaction of the electron magnetic moment and the positively charged ionic background gives rise to a "Spin Meissner" effect, the generation of a macroscopic spin current near the surface of superconductors. We point out that both the Meissner and the Spin Meissner effect can be understood dynamically under the assumption that the superfluid condensate wavefunction $\Psi(\vec{r})$ does not screen itself, just like the $\Psi(\vec{r})$ for an electron in a hydrogen atom. We argue that the conventional theory of superconductivity cannot explain the Meissner effect because it does not contain the physical elements discussed here.
\end{abstract}

PACS numbers:

\section{INTRODUCTION}

It is generally [1] believed that the conventional theory of superconductivity explains the Meissner effect [2], the telltale property of superconductors. This erroneous belief arises from two related widely held misconceptions. The first misconception is that the Meissner effect is an "equilibrium phenomenon" [3], that results from a particular form for the relation between current density and magnetic vector potential in the equilibrium state of a superconductor [4-6]. The second misconception is that for a theory of superconductivity to predict the Meissner effect it is sufficient that it yields a lower free energy for the system when the magnetic field is excluded than when the magnetic field remains inside the material [7].

The second misconception is easily dispelled by a counterexample. A superconducting ring with a finite current that generates an enclosed quantized magnetic flux has a higher free energy than the ring with no current and no magnetic flux. Yet there is no mechanism for the system to reach this lower energy state, and it will remain in the finite current "metastable" state forever.

The first misconception is also easily disproved. The Meissner effect is the process by which a metal cooled below its critical temperature in the presence of a magnetic field in its interior expels the magnetic field and reaches the equilibrium superconductive state with the magnetic field excluded. A calculation that deals only with quantities in the final state of this process, such as performed in Refs. [3-6] and many others cannot (by definition) prove that the system will reach this final state nor explain how the system does this. In the absence of a description of the process by which the equilibrium state is reached one can equally well conclude instead that the theory predicts that the system will forever remain in the initial "metastable" state, just like the ring discussed above.
Thus we argue that the current theory of superconductivity does not explain nor predict the Meissner effect. Rather, the most natural conclusion one should infer from the theory is that a magnetic field either in the interior of a ring, a hollow sphere or a solid body will remain unchanged (except perhaps for tiny adjustments to account for quantized flux) when the system is cooled below its critical temperature. That this should happen for all three cases was "proven" theoretically by Lippmann [8] in 1919 based on Faraday's law. It was confirmed experimentally by Onnes and Tuyn [9] in 1924 for the case of a hollow sphere. But it was disproved experimentally by Meissner and Ochsenfeld in 1933 [10] for the case of a solid body. We argue that how superconductors are able to prove Lippmann wrong and ignore Faraday's law is not explained by the conventional theory of superconductivity.

There has been a recent claim in the literature that the Meissner effect needs no explanation because any perfect conductor will show a Meissner effect [11]. echoeing a similar claim made earlier [12]. These claims are incorrect, as has been proven by several authors recently $[13,14]$ as well as earlier [15, 16]. For example, Ref. [14] shows that for the Meissner state to be energetically favorable requires the lowering of energy achieved by the superconducting condensation energy, which would not exist for a perfect conductor. While this certainly proves Ref. [11]'s claim wrong it does not prove that the Meissner effect is explained by the conventional theory for the reasons given above.

Why is the question whether or not the conventional theory of superconductivity explains the Meissner effect relevant? Because if the conventional theory cannot explain the Meissner effect it cannot be the correct theory of superconductivity, despite its many apparent successes. We argue that the conventional theory lacks essential physical ingredients that are necessary to explain 
the Meissner effect.

After discussing in more detail the absence of an explanation of the Meissner effect in the conventional theory in the next section, in the remainder of this paper we discuss what is required of a theory of superconductivity that can explain the Meissner effect. The theory of hole superconductivity contains those physical elements [17].

\section{CONVENTIONAL UNDERSTANDING OF THE MEISSNER EFFECT}

The London equation, that provides a phenomenological description of the Meissner effect, is usually made plausible [18-20] by starting from the 'acceleration equation' for a perfectly conducting fluid

$$
\frac{\partial \vec{v}_{s}}{\partial t}=\frac{q}{m} \vec{E}
$$

with $v_{s}$ the superfluid velocity for carriers of mass $m$ and charge $q$ and $\vec{E}$ the electric field, using Faraday's law

$$
\frac{\partial}{\partial t}\left(\vec{\nabla} \times \vec{v}_{s}\right)=-\frac{q}{m c} \frac{\partial \vec{B}}{\partial t}
$$

integrating Eq. (2) in time and setting the integration constant equal to zero to yield the London equation

$$
\vec{\nabla} \times \vec{v}_{s}=-\frac{q}{m c} \vec{B}
$$

with $\vec{B}$ the magnetic field. Combined with Maxwell's equation $\vec{\nabla} \times \vec{B}=(4 \pi / c) \vec{J}$, with $\vec{J}=n_{s} q \vec{v}_{s}$ the current density, Eq. (3) yields

$$
\nabla^{2} \vec{B}=\frac{4 \pi n_{s} q^{2}}{m c^{2}} \vec{B} \equiv \frac{1}{\lambda_{L}^{2}} \vec{B}
$$

describing the fact that a magnetic field cannot penetrate a superconductor beyond a distance $\lambda_{L}$ from the surface.

However, integrating Eq. (2) in time yields instead of Eq. (3)

$\vec{\nabla} \times \vec{v}_{s}(\vec{r}, t)-\vec{\nabla} \times \vec{v}_{s}(\vec{r}, t=0)=-\frac{q}{m c}(\vec{B}(\vec{r}, t)-\vec{B}(\vec{r}, t=0))$.

If a normal metal is cooled into the superconducting state in the presence of a spatially uniform magnetic field $\vec{B}(\vec{r}, t=0)=\vec{B}_{0}$ throughout its interior, the initial superfluid velocity $v_{s}(\vec{r}, t=0)=0$ and Eq. (5) yields

$$
\vec{\nabla} \times \vec{v}_{s}(\vec{r}, t)=-\frac{q}{m c}\left(\vec{B}(\vec{r}, t)-\vec{B}_{0}\right)
$$

which is not the same as Eq. (3). Quite the contrary, Eq. (6) implies that $\vec{v}_{s}(\vec{r}, t)=0$ and $\vec{B}(\vec{r}, t)=\vec{B}_{0}$ for all times $t>0$, so that the magnetic field remains unchanged inside the superconductor. Thus, this derivation certainly does not describe a process by which the system will achieve the Meissner state described by Eq. (3), rather it predicts that such a state will never be achieved.
Within the conventional (BCS) theory of superconductivity the Meissner effect is 'proven' [4-6] by calculating the London Kernel $K(\vec{q})$ relating the Fourier components of the current density $\vec{J}(\vec{q})$ and a static magnetic vector potential $\vec{A}(\vec{q})$

$$
\vec{J}(\vec{q})=-\frac{c}{4 \pi} K(\vec{q}) \vec{A}(\vec{q})
$$

and showing that

$$
K(q \rightarrow 0)=\frac{1}{\lambda_{L}^{2}} \neq 0
$$

when the system is described by the BCS wavefunction. Eq. (7) is equivalent to Eq. (3). A great deal of literature was generated around the question whether Eq. (7) could be proven in a gauge-invariant fashion, and eventually this was achieved to everyone's satisfaction [21]. However as discussed in the introduction this does not address the key question which is, how does the system achieve the BCS state that satisfies Eq. (7) starting from the initial state that doesn't?

Furthermore, there is an inherent problem in the linear response argument Eq. (7). It implicitly assumes that the system is initially in the BCS state, then a uniform magnetic field is created in its interior, and subsequently the system responds to it by generating the Meissner current that cancels the interior magnetic field. However it is physically impossible to 'create' a uniform magnetic field inside a material without magnetic lines 'cutting through' the material, since Maxwell's equations do not allow magnetic field lines to be created out of nowhere, they are always necessarily closed. If the system is initially in the BCS state it is a perfect conductor and magnetic field lines cannot cut through it so the linear response situation cannot be set up. In addition, a system that is already in the BCS state cannot have a magnetic field in its interior because this is incompatible with global phase coherence. What actually happens in the Meissner effect is that the system in the normal state is cooled below $T_{c}$, it is not in the BCS state initially, and in the process of entering the BCS state and establishing macroscopic phase coherence the magnetic field is expelled. This complex process is not described by Eq. (7).

A rationale for the conventional argument seems to be [22] that when the system is cooled below $T_{c}$ in the presence of a weak uniform magnetic field, it is expected that thermal fluctuations will lead to the transition into the superconducting state with the field expelled because it has lower free energy than the normal state. However, we argue that thermal fluctuations are local and such random fluctuations cannot generate the global surface current necessary to expel the magnetic field except with vanishing probability for a macroscopic system.

A somewhat more general 'proof' of the Meissner effect [23] starts from the assumption that the manyelectron superfluid condensate of a superconductor can 
be described by a complex macroscopic wavefunction $\Psi(\vec{r})$. This was first done in a phenomenological way by Ginzburg and Landau [24], later it was shown that such a description can be derived from BCS theory under certain conditions [25, 26], and the predictions of Josephson [27] and subsequent experimental verifications [28] established without doubt that it is a correct description of the superfluid condensate that captures the essence of superconductivity, whether BCS theory is valid or not. The amplitude of this macroscopic wave function $\Psi(\vec{r})$ is related to the density of superconducting carriers $n_{s}$

$$
\Psi(\vec{r})=|\Psi(\vec{r})| e^{i \phi(\vec{r})}=n_{s}^{1 / 2} e^{i \phi(\vec{r})}
$$

and the gradient of the phase $\phi(\vec{r})$ is related to the superfluid velocity $\vec{v}_{s}$ according to the relation

$$
\vec{v}_{s}=\frac{\hbar}{m} \vec{\nabla} \phi-\frac{q}{m c} \vec{A} .
$$

The wavefunction $\Psi(\vec{r})$ describes the state of all the Cooper pairs in the system, that share a common phase $\phi(\vec{r})$ that is coherent over macroscopic distances [28]. Many of the unique and universal properties of superconductors such as flux quantization and the variety of phenomena exhibited by Josephson junctions and 'weak links' follow from this simple macroscopic description. Upon taking the curl on both sides of Eq. (9) the London Eq. (3) results, hence this is assumed to be a proof of the Meissner effect. However once again, in assuming that the system is described by the macroscopic wavefunction Eq. (8) one is assuming that the magnetic field has already been expelled, since Eq. (8) is not valid in the presence of a magnetic field in the interior of the superconductor, and the process by which the system reaches or doesn't reach this state starting from the initial state with the magnetic field in the interior is not discussed.

Finally, an 'energetic' argument for the Meissner effect is given for example in ref. [7], where it is shown that the magnetic field distribution where the magnetic field is excluded except in a region within $\lambda_{L}$ of the surface minimizes the free energy. However as already emphasized, this does not explain the process of field expulsion nor predicts that the state will be reached.

In summary, we argue that these arguments, which are generally assumed to prove that the conventional theory of superconductivity describes the Meissner effect, in fact do not do so, and leave completely open the question whether or not the conventional theory of superconductivity can describe the Meissner effect.

More recently, there have been calculations describing the normal-superconductor transition in a magnetic field $[29,30]$ using time-dependent Ginzburg Landau theory $[31,32]$. In TDGL theory it is assumed that the superconducting order parameter $(\Psi(\vec{r}))$ relaxes exponentially to its equilibrium value in a non-equilibrium situation. However, no justification for this assumption is presented. In fact, very recent work claims [33, 34] that this assumption of TDGL theory is incorrect and that within BCS-Bogoliubov-de-Gennes theory the superconducting order parameter will not relax spontaneously to its equilibrium value.

\section{HOW A PERFECT CONDUCTOR EXPELS A MAGNETIC FIELD}

Let us consider again the equation of motion for a perfectly conducting fluid. Eq. (1) is not quite correct because it ignores the difference between total and partial time derivatives, and because the magnetic Lorentz force is omitted from the right side. Taking both of these facts into account leads instead of to Eq. (2) to the equation [35]

$$
\frac{\partial \vec{w}}{\partial t}=\vec{\nabla} \times\left(\overrightarrow{v_{s}} \times \vec{w}\right)
$$

with

$$
\vec{w}=\vec{\nabla} \times \overrightarrow{v_{s}}+\frac{q}{m c} \vec{B}
$$

the 'generalized vorticity'. The London Eq. (3) is

$$
\vec{w}(\vec{r}, t)=0
$$

and the initial condition at the moment a system is cooled into the superconducting state in a uniform magnetic field $B_{0}$ is

$$
\vec{w}(\vec{r}, t=0)=\vec{w}_{0}=\frac{q}{m c} \vec{B}_{0} .
$$

To explain the Meissner effect along these lines one has to explain how $\vec{w}$ evolves in time from its initial value Eq. (12) to its final value Eq. (11) at all $\vec{r}$ s at a later time.

Let us assume for simplicity a cylindrical geometry with $\vec{B}$ in the $z$ direction. $\vec{w}=w \hat{z}$ and Eq. (10a) can be rewritten as

$$
\frac{\partial w}{\partial t}=-\frac{1}{r} \frac{\partial}{\partial r}\left(r w v_{r}\right)
$$

which indicates that a radial velocity $v_{r} \neq 0$ of the fluid is a prerequisite for $w$ to change in time. In the absence of a radial velocity, $\partial w / \partial t=0$ and $w(\vec{r}, t)=w_{0}$ for all times and the magnetic field in the interior of the metal becoming superconducting would remain unchanged, in agreement with Lippmann [8] and in contradiction with experiment.

Eq. (13) can be rewritten as

$$
\frac{\partial w}{\partial t}=-\vec{\nabla} \cdot\left(w \overrightarrow{v_{s}}\right)
$$

which is a continuity equation. It says that for $w$ to change locally it has to be carried away by the fluid flow. Integrating Eq. (14) over a cylinder of radius $\mathrm{R}$ and using Gauss' theorem yields

$$
\int_{0}^{R} d r r \frac{\partial}{\partial t} w(r, t)=-R w(R, t) v_{r}(R, t)
$$


and integrating over time and assuming at time $\mathrm{T}$ the Meissner state $w(r, T)=0$ for all $r \leq R$ has been reached yields the condition

$$
w_{0}=\frac{2}{R} \int_{0}^{T} d t w(R, t) v_{r}(R, t) .
$$

According to this calculation in order to achieve the Meissner state in the interior of a cylindrical superconductor of radius $R$, i.e. $w(r)=0$ for all $r \leq R$, the entire superfluid has to flow out of the material carrying the generalized vorticity $w$ with it.

Of course superconductors don't do that. The reason this calculation does not apply is that it assumes the system first becomes a perfect conductor and subsequently expels the magnetic field As we discuss in the next section, this is not what occurs in reality. Nevertheless this analysis is useful because it indicates that an outflow of charge is necessary to understand the dynamics of the Meissner effect.

\section{HOW THE TRANSITION OCCURS}

In a recent paper [36] we have discussed the kinetics of the normal-superconductor transition in a magnetic field. Figure 1 shows three generic scenarios. As discussed in [36], the simplest scenario (a), where a current develops near the surface of the sample and the magnetic field is uniformly expelled can be excluded on theoretical as well as experimental grounds. In scenario (b) superconducting regions nucleate at random and expand. Scenarios (b) and (c) are in essence similar so we will limit ourselves to scenario (c), where the superconducting phase expands from a nucleus at the center towards the surface with a surface current that excludes the magnetic field in the interior of the superconducting region. This has been implemented experimentally in a cylinder by applying a slightly smaller magnetic field in the central region [37].

Both in scenarios (b) and (c) a Faraday electric field $E$ exists at and near the boundary of the domains because the magnetic field is changing as the domains expand. The direction of this electric field is opposite to the direction of the boundary current as shown in Fig. 1(c), to induce a current opposing the change in magnetic flux (Lenz's law). Such a counterclockwise electric field also exists throughout the interior of the sample in (a) during the transition.

Focusing on scenario (c), an explanation of the Meissner effect has to explain how this current at the surface of the expanding superconducting region is generated in the first place, how it is maintained on the face of the Faraday electric field that tries to suppress it, how its momentum and kinetic energy is transferred radially outward as the superconducting phase expands, and how this growing momentum (and angular momentum) is compensated so as to not violate fundamental conservation laws. None of these questions is addressed by theoretical treatments

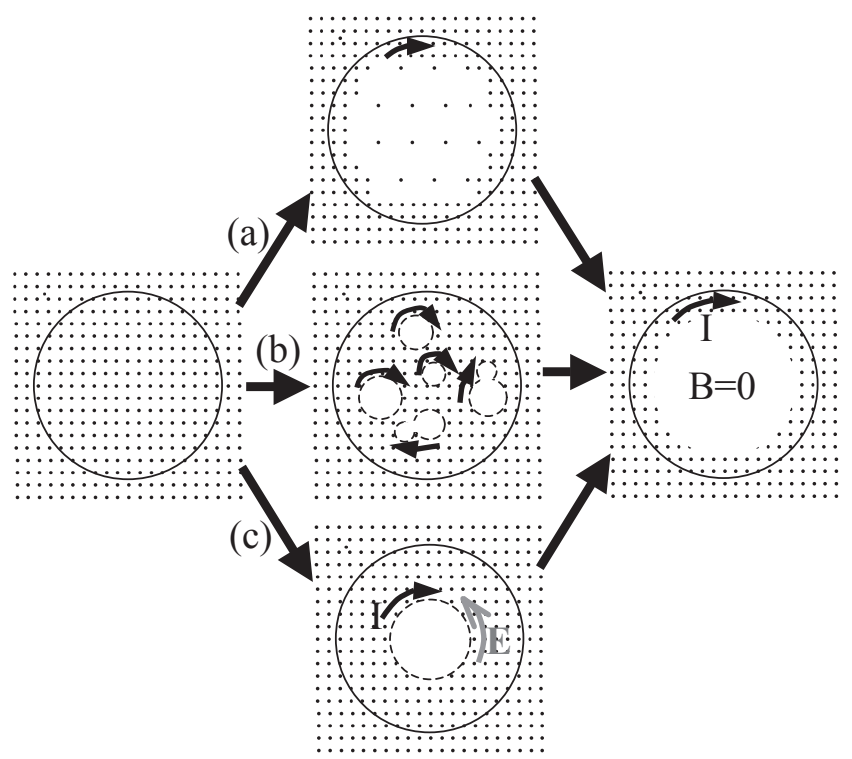

FIG. 1: Three conceivable routes for the magnetic field expulsion in a cylindrical superconductor. The dots represent magnetic field lines coming out of the paper. The arrows give the direction of the currents $(I)$. In (a), a current starts developing within $\lambda_{L}$ of the surface of the cylinder that gradually increases in magnitude, causing a gradual uniform decrease of the magnetic field in the interior. In (b), domains of loop currents start at various random locations that nullify the magnetic fields in their interior, that gradually expand their radii and coalesce and merge with neighboring domains. In (c), a single circular domain starts at the center and expands in radius until it reaches the boundary of the cylinder.

of this process based on the conventional BCS-LondonTDGL theory of superconductivity [29, 30].

Figure 2 (a) shows more stages of the process of magnetic field expulsion (c) of Fig. 1. BCS-London-TDGL theory would say that the physics driving the expansion of the field-free region is the superconducting condensation energy. The energy of the system is lowered as Cooper pairs form and become phase coherent, condensing into the macroscopic superconducting state described by $\Psi(\vec{r})$. Because the establishment of phase coherence requires that no magnetic field exists in that region, the region has to become field free, and this occurs through a BCS order parameter spontaneously growing and relaxing towards its equilibrium value $[29,30]$. This is a phenomenological treatment that does not explain how the condensation into the BCS state is connected to the azimuthal motion of the carriers that needs to be generated at the boundary, and in particular what is the force that drives these carriers to move in direction opposite to the electric force exerted on them by the Faraday electric field. Within BCS-London-TDGL theory there is no radial charge motion associated with the outward motion of the phase boundary between superconducting and normal regions.

The behavior shown in Fig. 2 (a) would also occur if 
(a) Growth of superconducting phase
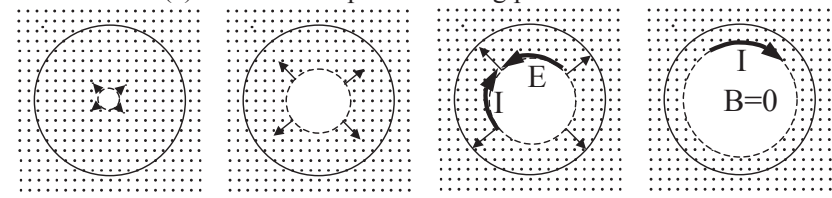

(b) Outward flow of perfectly conducting fluid
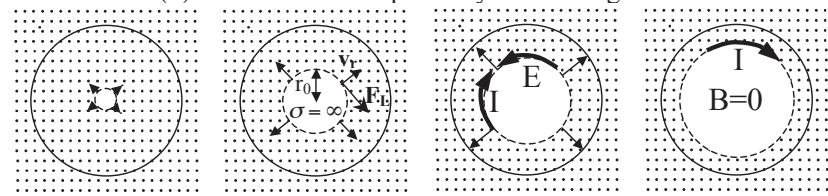

FIG. 2: (a) More stages of the process of magnetic field expulsion as a system goes superconducting according to the scenario (c) of Fig. 1. At and near the boundary of the expanding field-free region there is an electric field pointing counterclockwise, due to the changing magnetic flux and Faraday's law, and a current flowing clockwise that cancels the applied magnetic field in the interior. (b) Schematic depiction of a perfectly conducting fluid $(\sigma=\infty)$ that flows radially outward with radial velocity $v_{r}$. The carriers at the boundary experience a Lorentz force. Assuming the sign of the charge $q$ is positive for definiteness the Lorentz force $F_{L}=(q / c) v_{r} B$ points in the clockwise direction. The resulting electric current $I$ at the boundary flows clockwise (for negative charge carriers the Lorentz force would be in opposite direction, the current in the same direction), generating a magnetic field opposite to the external field so that no magnetic field penetrates the perfect conductor.

a core of high density perfectly conducting material (infinite conductivity) would expand radially outward, as shown schematically in Fig. 2 (b). Here the charge carriers are moving radially out and there is a Lorentz force $F_{L}$ acting on them giving them an azimuthal velocity (in the clockwise direction assuming positive carriers) such that they generate a magnetic field opposite to the applied one, thus not allowing the magnetic field to penetrate the interior of the perfectly conducting region (except to within a distance $\lambda_{L}$ of its surface). We discuss the process quantitatively in what follows in a planar geometry for simplicity.

\section{MEISSNER EFFECT IN PLANAR GEOMETRY}

Figure 3 shows the processes of Fig. 2 in a planar geometry. The magnetic field points in the $z$ direction, the phase boundary advances in the $+x$ direction, and the Faraday electric field and Meissner current point in the $y$ direction. $x_{0}(t)$ is the boundary of the superconducting region, moving upward at rate $d x_{0} / d t$. The magnetic and electric fields for $x \leq x_{0}$ are given by

$$
B(x)=B_{0} e^{\left(x-x_{0}\right) / \lambda_{L}}
$$
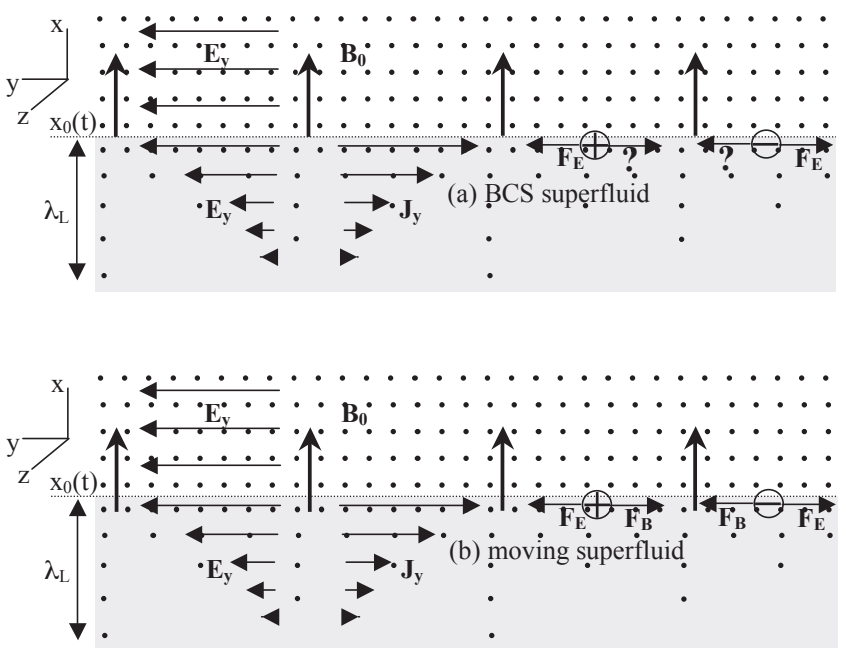

FIG. 3: Magnetic field $B_{0}$ points out of the paper. (a) Superconducting-normal phase boundary $x_{0}(t)$ moving in the $+x$ direction with no associated charge flow in the $x$ direction. (b) Superfluid moving in the $+x$ direction with boundary at $x_{0}(t)$. For both cases the magnetic field is expelled from the region $x<x_{0}(t)$ by the current $J_{y}$ flowing within $\lambda_{L}$ of the boundary, and a Faraday field $E_{y}$ exists due to the changing magnetic field. The electric Lorentz force $\left(F_{E}\right)$ drives the carriers in direction opposite to their direction of motion. Only for case (b) the carriers also experience a magnetic Lorentz force $F_{B}$ driving them in the direction of their motion, opposite to $F_{E}$ and of equal magnitude.

$$
E_{y}(x)=\frac{B_{0}}{c} \frac{d x_{0}}{d t} e^{\left(x-x_{0}\right) / \lambda_{L}}
$$

with $B_{0} \hat{z}$ the applied magnetic field and $E_{y}$ in the positive $y$ direction. Assuming current carriers of charge $q$, mass $m$ and density $n_{s}$, their speed in the $y$ direction is

$$
v_{y}(x)=-\frac{c}{4 \pi n_{s} q \lambda_{L}} B_{0} e^{\left(x-x_{0}\right) / \lambda_{L}}
$$

parallel (antiparallel) to the current, which flows in the $-\hat{y}$ direction, for $q>0(q<0)$. For $x>x_{0}$ we assume for simplicity

$$
B(x)=B_{0}
$$

$$
E_{y}(x)=\frac{B_{0}}{c} \frac{d x_{0}}{d t}
$$

This assumption corresponds to the treatment of ref. [36] for the particular case where the magnetic field that is being expelled is very close to the critical field $(p \rightarrow 0$ in the notation of ref. [36]).

Equations $(17 \mathrm{a}, \mathrm{b}, \mathrm{c})$ follow from the relation between current and velocity $\vec{J}=n_{s} q \vec{v}$, the expression for the London penetration depth Eq. (4), Maxwell's equations

$$
\vec{\nabla} \times \vec{B}=\frac{4 \pi}{c} \vec{J}==>\frac{\partial B}{\partial x}=-\frac{4 \pi}{c} J_{y}=-\frac{4 \pi n_{s} q}{c} v_{y}
$$




$$
\vec{\nabla} \times \vec{E}=-\frac{1}{c} \frac{\partial \vec{B}}{\partial t}==>\frac{\partial E_{y}}{\partial x}=-\frac{1}{c} \frac{\partial B}{\partial t}
$$

and London's equation

$$
\vec{\nabla} \times \vec{J}=-\frac{n_{s} q^{2}}{m c} \vec{B}==>\frac{\partial v_{y}}{\partial x}=-\frac{q}{m c} B .
$$

The electromagnetic force acting on the carriers is

$$
m \frac{d \vec{v}}{d t}=\frac{q}{m} \vec{E}+\frac{q}{m c} \vec{v} \times \vec{B}
$$

and its component in the $y$ direction yields

$$
\frac{\partial v_{y}}{\partial t}+v_{x} \frac{\partial v_{y}}{\partial x}=\frac{q}{m} E_{y}-\frac{q}{m c} v_{x} B
$$

For the case of Fig. 3(b) (moving superfluid) we have

$$
v_{x}=\frac{d x_{0}}{d t}
$$

and the terms on the right-hand side of Eq. (21) satisfy

$$
F_{E}=\frac{q}{m} E_{y}=\frac{q}{m c} v_{x} B=F_{B}
$$

so that the electric and magnetic forces in Eq. (21) exactly cancel out. The left-hand side of Eq. (21) is also identically zero from Eqs. (17c) and (22). Carriers are accelerated by the magnetic Lorentz force and decelerated by the electric force arising from Faraday's field, which exactly cancel in steady state. Thus, a dynamical explanation of flux expulsion is provided by these equations: the magnetic Lorentz force acting on the outflowing charge drives the current that nullifies the magnetic field in the interior against the Faraday electric force.

For the processes in Figs. 3 to happen requires a driving force in the $+\hat{x}$ direction. The way this works is very clear for case (b), moving superfluid: there is a magnetic Lorentz force on the $J_{y}$ current carriers in the $-\hat{x}$ direction:

$$
F_{x}=\frac{q}{c}(\vec{v} \times \vec{B})_{x}=\frac{q}{c} v_{y} B
$$

so that an equal and opposite force needs to be applied in the $+\hat{x}$ direction for the superfluid to advance in the $+\hat{x}$ direction. This requires expenditure of energy per unit time per carrier $-F_{x} v_{x}$, so that the energy per unit area per unit time spent is, from Eqs. (17a), (17c) and (24)

$$
\int_{-\infty}^{x_{0}} d x n_{s}\left(-F_{x} v_{x}\right)=\int_{-\infty}^{x_{0}} d x \frac{B(x)^{2}}{4 \pi \lambda_{L}} v_{x}=\frac{B_{0}^{2}}{8 \pi} \frac{d x_{0}}{d t}
$$

which equals the rate of change of magnetic energy per unit area as the boundary moves, as required by energy conservation. There is no energy dissipated in this process.

The same rate of energy expenditure is required for the process of Figure 3(a) to expel the magnetic field. This energy obviously is supplied by the superconducting condensation energy. However since in this case $v_{x}=0$, Eq. (25) cannot be used to understand how the condensation energy causes the phase boundary to advance. The force $F_{x}$ given by Eq. (24) still exists in this case, but since carriers don't move in the $x$ direction this force does not do any work. The work done by the condensation process in moving the phase boundary in the $+\hat{x}$ direction without displacing charge carriers in the $+\hat{x}$ direction occurs through some unknown way.

In addition since charge does not move in the direction of the moving phase boundary there is no magnetic Lorentz force driving the Meissner current in Fig. 3(a) and Eq. (21) is not satisfied. Eq. (21) becomes

$$
\frac{\partial v_{y}}{\partial t}=\frac{q}{m} E_{y}
$$

which certainly does not describe Fig. 3(a) since it says that the current should be flowing in the opposite direction, parallel rather than antiparallel to $E_{y}$. In steady state the left side in Eq. (26) is zero and there is an uncompensated right-hand-side.

One would have to include other dynamical effects to 'fix' Eq. (21) and to give a dynamical explanation of the energy transfer process for the case of Fig. 3(a) other than Eq. (25). This has not been done within the conventional theory. We argue that the dynamics described by Eqs. (20) and (25), requiring charge motion in the same direction as the phase boundary motion, is the only physical way to describe the flux expulsion.

\section{BACKFLOW}

As discussed in the previous section, the strong similarity in the physics shown in Figs. 3(a) and 3(b) is compelling evidence that in the process of a metal becoming superconducting an outflow of charge in direction normal to the superconductor-normal metal phase boundary into the normal region takes place. This explains both how the carriers in the supercurrent flowing parallel to the phase boundary can move in direction opposite to the Faraday electric force, and how the superconducting condensation energy is used to do the work required to expel the magnetic field: the condensation results in an electromotive force [38] driving the carriers to move in the $+\hat{x}$ direction, i.e. from the superconducting into the normal region, opposing the electromagnetic force Eq. 24.

In order for this not to create an enormous charge imbalance it requires a backflow of normal carriers in the $-\hat{x}$ direction. Figure 4 shows schematically how this can happen. The layer of superfluid of thickness $\lambda_{L}$ next to the phase boundary with the velocity pattern given by Eq. (17c) moves forward a distance $\lambda_{L}$, and an equal amount of normal fluid moves backward, and in the process becomes superfluid. This satisfies energy and momentum 


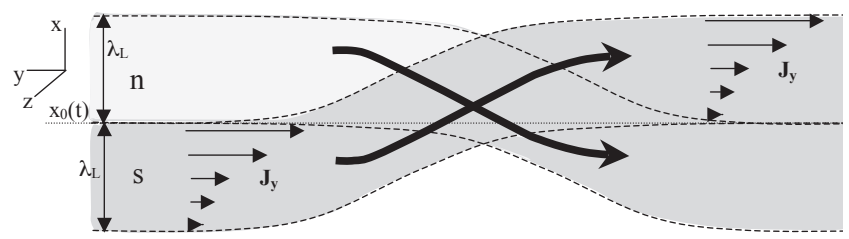

FIG. 4: Schematic depiction of how the superfluid-normal phase boundary moves into the normal region. Superfluid layer of thickness $\lambda_{L}$ carrying the screening current $\vec{J}_{y}$ moves forward and an equal amount of normal fluid moves backward and becomes superconducting.

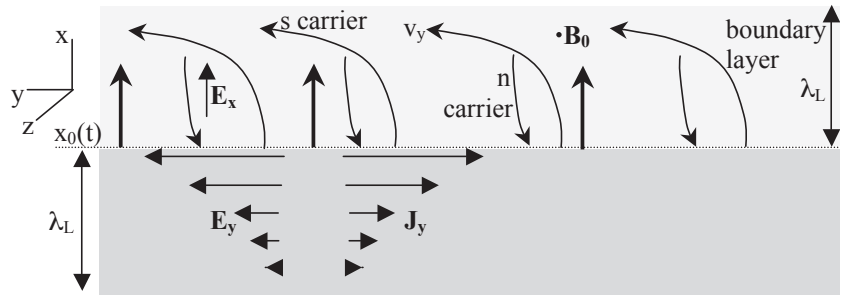

FIG. 5: As carriers become superconducting (s carriers) they thrust forward into the normal region over a boundary layer of thickness $\lambda_{L}$, and are deflected by the Lorentz force acquiring speed $v_{y}=-c /\left(4 \pi n_{s} q \lambda_{L}\right) B_{0}$. This process creates an electric field $E_{x}$ in the $-\hat{x}$ direction that drives normal carrier (n carrier) backflow. Here, "n" stands both for "normal" and "negative". The normal carriers do not acquire a large $v_{y}$ in opposite direction because they scatter off impurities and transfer their $y$-momentum to the lattice.

conservation and gives a simple description of the process. The driving force for the backflow is the electric field in direction perpendicular to the phase boundary created by the forward flow.

However, considered in detail the process is likely to be more complicated. We propose that the forward motion occurs for carriers that are in the process of becoming superconducting, which are initially (essentially) not moving in the $y$ direction and acquire a large velocity parallel to the phase boundary through the magnetic Lorentz force. As discussed in Ref. [36], in order for a carrier moving in the $+\hat{x}$ direction to acquire the transverse velocity Eq. (17c) through the action of the magnetic Lorentz force requires a displacement over a distance $\lambda_{L}$ in the $x$ direction. Thus, we envision a process of forward flow and backflow over a boundary layer of thickness $\lambda_{L}$ as the phase boundary advances, as shown schematically in Fig. 5. As carriers become superconducting they thrust forward a distance $\lambda_{L}$, which creates an electric field in the $+\hat{x}$ direction (assuming these are negatively charged carriers) causing backflow of normal carriers in this layer. As the phase boundary advances at rate $v_{0}=d x_{0} / d t$, normal (negatively charged) carriers in the boundary layer are back-flowing at speed $v_{0}$, or equivalently positive normal carriers (holes) move forward together with the phase boundary. Because normal carriers scatter off the lattice they do not acquire a

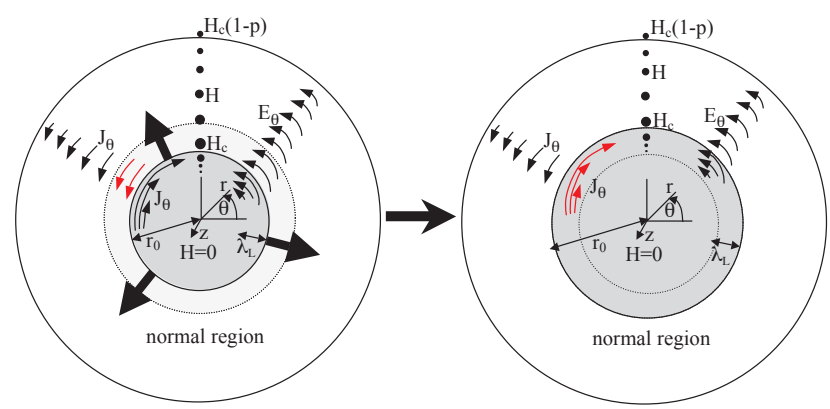

FIG. 6: Expansion of superconducting phase in a cylindrical geometry. On the left, the dark grey circle is superconducting and the light grey ring (of thickness $\sim \lambda_{L}$ ) is about to become superconducting. As the ring becomes superconducting the current direction in the ring switches from the $+\hat{\theta}$ to the $-\hat{\theta}$ direction (red arrows) which is opposite to the electric field direction.

large $v_{y}$ from the action of the magnetic Lorentz force, instead they transfer their $y$-momentum to the lattice as a whole, thus accounting for momentum conservation.

This gives a phenomenological description of the magnetic field expulsion process that resolves the puzzles of the Meissner effect regarding forces and momentum conservation. A complete microscopic description has not yet been proposed. A semiclassical explanation based on the proposal that superconducting carriers reside in mesoscopic orbits of radius $2 \lambda_{L}$ [39] is discussed in the following sections.

For completeness we show in figure 6 the current and fields in a cylindrical geometry. The magnetic field that is being expelled is $H_{c}(1-p)$ with $p>0$, and it increases to $H_{c}$ at the phase boundary due to the magnetic field generated by the eddy current in the normal region. The equations are given in Ref. [36]. Again, the process is simply understood assuming the outward expansion of the phase boundary is accompanied by radial outflow of charge, driven by a radial electromotive force that pushes the charges against a radially inward pointing magnetic Lorentz force. An inward backflow of normal current (not shown) compensates for the charge imbalance. In the absence of radial outflow of charge there is no mechanism to explain how the current reverses its azimuthal direction (red arrows) and flows against the direction of the electric field when the phase boundary advances, nor to explain how the growing angular momentum of the Meissner current is compensated [36, 42].

\section{MEISSNER EFFECT AND $2 \lambda_{L}$ ORBITS}

Consider a superconducting long cylinder in an applied magnetic field $H$. The Meissner current that nullifies the magnetic field in the interior flows within a distance $\lambda_{L}$ 
of the surface with speed

$$
v_{s}=-\frac{e \lambda_{L}}{m_{e} c} H .
$$

with $e$ and $m_{e}$ the electron charge and mass. This is easily seen from the relation between magnetic field and vector potential for the cylinder, $A=H \lambda_{L}$. The Meissner current density is

$$
j_{s}=n_{s} e v_{s}=\frac{n_{s} e^{2}}{m_{e} c} \lambda_{L} H
$$

and from Maxwell's equation $\vec{\nabla} \times \vec{H}=(4 \pi / c) \vec{j}_{s}$ it follows that

$$
H=\frac{4 \pi}{c} j_{s} \lambda_{L}
$$

and combining Eqs. (28) and (29) yields

$$
\frac{1}{\lambda_{L}^{2}}=\frac{4 \pi n_{s} e^{2}}{m_{e} c^{2}} .
$$

The magnetization per unit volume is given by

$$
M=\frac{H}{4 \pi}=\frac{j_{s}}{c} \lambda_{L}=\frac{e n_{s}}{c} \lambda_{L} v_{s}
$$

It is reasonable to assume that the magnetization results from a superposition of elementary magnetic moments $\vec{\mu}$ resulting from the orbital motion of each superconducting electron:

$$
\vec{M}=n_{s} \vec{\mu}
$$

This yields for the magnetic moments

$$
\mu=\frac{e \lambda_{L}}{c} v_{s}
$$

and from the general principle that the magnetic moment and orbital angular momentum $\vec{\ell}$ of each electron are related by

$$
\vec{\mu}=\frac{e}{2 m_{e} c} \vec{\ell}
$$

we deduce that the angular momentum associated with each of these electrons is

$$
\ell=\frac{2 m_{e} c}{e} \mu=m_{e} v_{s}\left(2 \lambda_{L}\right) .
$$

Eq. (35) implies that the Meissner current that nullifies the magnetic field in the interior of a superconductor results from each superfluid carrier moving with speed $v_{s}$ in a mesoscopic orbit of radius $2 \lambda_{L}$. Just like in a magnetic material, the superposition of elementary currents results in a macroscopic surface current.

It can be seen that the fact that electrons reside in $2 \lambda_{L}$ orbits by itself is sufficient to give perfect diamagnetism, as follows. For an electron in an orbit of radius $r$, application of an external field $H$ yields through Faraday's law a tangential electric field

$$
E=-\frac{r}{2 c} \frac{\partial H}{\partial t}
$$

and from the equation of motion $m_{e} d v / d t=e E$,

$$
\frac{d v}{d t}=-\frac{e r}{2 m_{e} c} \frac{\partial H}{\partial t}
$$

so that Eq. (27) results upon integration for $r=2 \lambda_{L}$. However there is a subtle question of self-consistency that we discuss in the next section.

\section{MEISSNER EFFECT AND MAGNETIC SUSCEPTIBILITY}

An external magnetic field $H$ applied to a material gives rise to a magnetization $M=\chi H$ and a total magnetic field

$$
B=H+4 \pi M=(1+4 \pi \chi) H .
$$

A perfect diamagnet does not allow any magnetic field in its interior, hence is defined by $\chi=-1 /(4 \pi)$ according to Eq. (38). This is usually assumed to be the susceptibility of superconductors.

However, Pippard points out [40] that this is a misconception when applied to superconductors because "the mean field responsible for magnetizing an extended unit is not $H$ but $B$ ", from which it follows that $M=\chi B$ rather than $M=\chi H$ in Eq. (38), hence

$$
B=\frac{1}{1-4 \pi \chi} H,
$$

and "perfect diamagnetism demands that $\chi$ be infinitely negative" [40]. The same argument is made by Tinkham [41].

Consider a system of electrons of density $n_{s}$ per unit volume in orbits perpendicular to an applied magnetic field $H$. The Larmor diamagnetic susceptibility is given by

$$
\chi=-\frac{n_{s} e^{2}}{4 m_{e} c^{2}}<r^{2}>
$$

where $\sqrt{\left.<r^{2}\right\rangle} \equiv \bar{r}$ gives the spatial extent of the orbit, i.e. its 'radius'. For

$$
\bar{r}=2 \lambda_{L}
$$

the diamagnetic susceptibility Eq. (40) is $\chi=-1 / 4 \pi$ according to Eq. (30), and we have argued in the past that this should apply to superconductors [42]. Instead, Pippard and Tinkham argue, as discussed above, that $\bar{r}=\infty$ is required in superconductors to give $\chi=-\infty$ and $B=0$ (Eq. (39)). 
Indeed it would appear at first sight that the PippardTinkham point of view is the correct one. The orbits of radius given by Eq. (41) are highly overlapping since

$$
2 \lambda_{L}=n_{s}^{-1 / 3} \sqrt{\frac{n_{s}^{-1 / 3}}{\pi r_{c}}}>>n_{s}^{-1 / 3}
$$

where $r_{c}=e^{2} / m_{e} c^{2}$ is the classical electron radius, much smaller than the inter-electron distance $n_{s}^{-1 / 3}$. Therefore one would expect the magnetic field generated by one orbit to reduce the magnetic field affecting nearby overlapping orbits, and the net magnetic field resulting from application of an external magnetic field $H$ to be, from Eq. (39)

$$
B=\frac{1}{1-4 \pi(-1 / 4 \pi)} H=\frac{H}{2}
$$

which is not the complete flux expulsion observed in superconductors.

The same result is obtained from the analysis in the previous section. Faraday's law Eq. (36) should involve the total field $B$ rather than $H$, hence instead of Eq. (37) we have

$$
\frac{d v}{d t}=-\frac{e r}{2 m_{e} c} \frac{\partial B}{\partial t}
$$

The total magnetic field is weaker than the applied magnetic field due to the counterfield generated by the other electrons in the system:

$$
B=H+4 \pi M=H+4 \pi n_{s} \mu=H-\frac{2 \pi n_{s} e r}{c} v
$$

using that $\mu=-e r v / 2 c$ for electrons with speed $v$ in orbits of radius $r$. Replacing in Eq. (44) and performing the time integration yields

$$
v=-\frac{\frac{e r}{2 m_{e} c}}{1+\frac{\pi n_{s} e^{2}}{m_{e} c^{2}} r^{2}} H
$$

which for $r=2 \lambda_{L}$ yields

$$
v=-\frac{e \lambda_{L}}{2 m_{e} c} H=\frac{v_{s}}{2} .
$$

predicting that electrons aquire only half of the required speed Eq. (27) to nullify the applied magnetic field $H$, hence that the applied magnetic field will not be nullified in the interior but only reduced by a factor of 2 , in agreement with Eq. (43).

This result is puzzling because a perfect classical conductor will perfectly screen an applied magnetic field in its interior. We conclude that to make this semiclassical model agree with the classical behavior requires that Eq. (37) rather than Eq. (44) applies, in other words that electrons in the superfluid are only affected by the external magnetic field and not by the magnetic field generated by other electrons in the superfluid.
Thus, to understand the perfect diamagnetism of superconductors in terms of $2 \lambda_{L}$ orbits as suggested by Eqs. (32) and (35) it is necessary to assume that the magnetic field created by superfluid electrons does not affect the superfluid itself. This is not an implausible assumption. It will occur if the superconducting electrons are described by a macroscopic wavefunction $\Psi(\vec{r})$ that does not screen itself. Thus, when an external magnetic field is applied all the components of the macroscopic wavefunction are subject to the entire applied field rather than to the external field reduced by the action of other components of the wavefunction. Under those conditions, Eq. (37) rather than Eq. (44) applies and the induced magnetic field for electrons in orbits of radius $2 \lambda_{L}$ is precisely of the magnitude needed to completely cancel the interior magnetic field.

This key property of the quantum-mechanical wave function was recognized from the outset of the development of quantum mechanics. Schrödinger expressed it clearly in his 1952 paper on the meaning of wave mechanics [43]: "The original wave mechanical model of the hydrogen atom is not self-consistent. The electronic cloud effectively shields the nuclear charge towards outside, making up a neutral whole, but is inefficient inside; in computing its structure its own field that it will produce must not be taken into account, only the field of the nucleus." The reason Schrödinger spelled this out in detail is because it contradicted his intuition and physical expectation that $|\Psi(\vec{r})|^{2}$ would represent the charge density of the electron at position $\vec{r}$ rather than the probability of finding the electron at $\vec{r}$.

As we discuss in the next section, the same physics explains how a magnetic field is expelled from the interior of a system becoming superconducting, and furthermore we argue that this process cannot be explained in the absence of this physics.

\section{DYNAMICAL EXPLANATION OF THE MEISSNER EFFECT}

The Landau diamagnetic susceptibility of conduction electrons is given by

$$
\chi=-\frac{1}{3} \mu_{B} g\left(\epsilon_{F}\right)
$$

with $g\left(\epsilon_{F}\right)$ the density of states at the Fermi energy and $\mu_{B}$ the Bohr magneton. Using the expression for the free electron density of states $g\left(\epsilon_{F}\right)=3 n / 2 \epsilon_{F}$ it is found that the Landau susceptibility Eq. (48) is the Larmor diamagnetic susceptibility Eq. (40) for

$$
<r^{2}>=k_{F}^{-2} \text {. }
$$

and $n=n_{s}$, hence that in the normal state electrons reside in microscopic non-overlapping orbits of radius $k_{F}^{-1}$. Therefore, in the process of becoming superconducting and establishing phase coherence electrons expand their orbits from radius $k_{F}^{-1}$ to radius $2 \lambda_{L}$. 


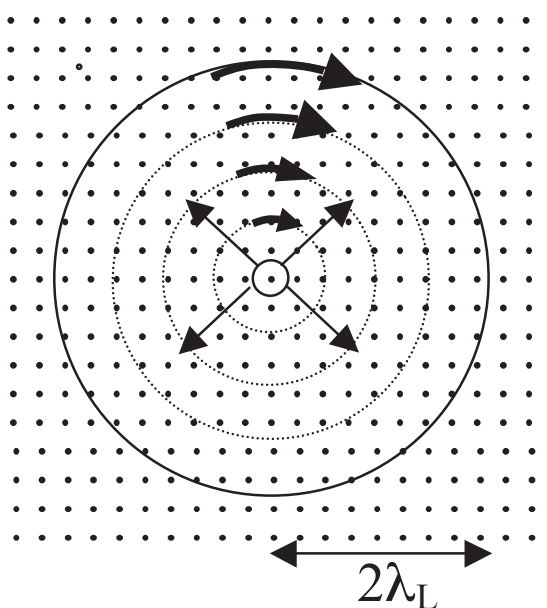

FIG. 7: A single charge carrier of positive charge expanding its orbit in a magnetic field pointing out of the paper acquires an azimuthal velocity in the clockwise direction, and itself generates a (small) magnetic field pointing into the paper.

The process of expansion of electronic orbits from radius $k_{F}^{-1}$ to radius $2 \lambda_{L}$ provides a dynamical explanation of the Meissner effect. Due to the action of the Lorentz force on the radially outmoving electron it acquires an azimuthal velocity given by [44]

$$
v_{\theta}=-\frac{q r}{2 m c} H
$$

which is identical to Eq. (37), the speed acquired by a charge in an orbit of radius $r$ when the applied magnetic field is increased from 0 to its final value $H$. The reason is Faraday's law for Eq. (37), Lorentz force for Eq. (50). When the radial motion is over a distance $r=2 \lambda_{L}$ the azimuthal velocity acquired is

$$
v_{\theta}=-\frac{q \lambda_{L}}{m c} H
$$

which is the same as the speed of the Cooper pairs in the Meissner current Eq. (27). Note that it is important in this analysis that the magnetic field imparting the azimuthal velocity to the electron in the expanding orbit is $H$ rather than $B$.

Figure 7 shows schematically the expansion of a single orbit in a magnetic field pointing out of the paper. As the orbit expands the carrier's orbit cuts through magnetic field lines and in so doing acquires an azimuthal velocity, shown clockwise in Fig. 7 assuming the carrier has positive charge. When the radius reaches $2 \lambda_{L}$, the azimuthal speed reaches the value Eq. (27). In turn, the motion of this charge generates a magnetic field in the direction opposite to the applied field, i.e. into the paper in Fig. 7. For a single carrier of course the magnitude of this counterfield is negligible.

Now we consider the growth of the superconducting region in a cylinder. In the normal outer region the orbits are microscopic, in the interior superconducting

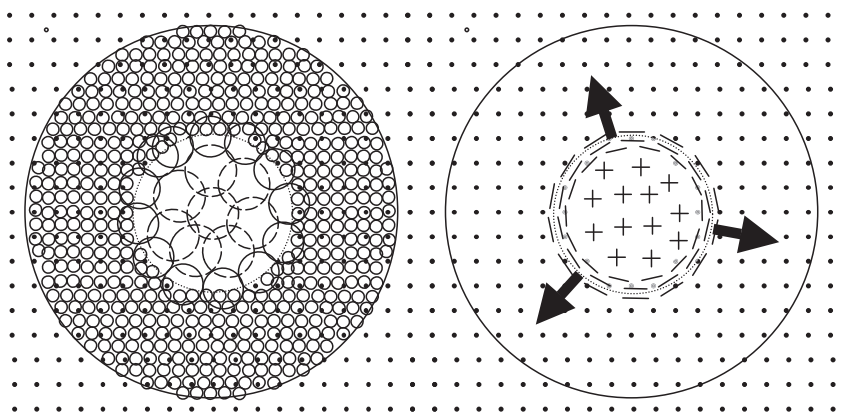

FIG. 8: Left panel: In the superconducting region the carriers reside in orbits of radius $2 \lambda_{L}$, in the normal region in orbits of radius $k_{F}^{-1}$. At the boundary of the normal-superconducting region (dotted circle) the orbits expand from radius $k_{F}^{-1}$ to radius $2 \lambda_{L}$, causing magnetic field expulsion. Right panel: in the superconducting region the charge distribution is not homogeneous, there is an excess negative charge within $\lambda_{l}$ of the surface of the superconducting region that spills over into the normal region, and an excess positive charge in its interior.

region the orbits have radius $2 \lambda_{L}$, and at the boundary between superconducting and normal regions the orbits are expanding. This is shown schematically in Figure 8 (left panel). The carrier in each expanding orbit reaches the final azimuthal velocity Eq. (27) when the radius of the orbit reaches its final value $2 \lambda_{L}$. Superposition of these motions results in a current being carried within a layer of thickness $\lambda_{L}$ from the boundary of the normal-superconducting region. As the superconducting region expands, this boundary current expands with the boundary, and no net current remains in the interior region due to cancellation of the internal motions. When the boundary reaches the boundary of the sample, the system reaches the superconducting state where all the magnetic field has been excluded except within a layer of depth $\lambda_{L}$ from the surface, and a Meissner current flows in this layer. In this process it is crucial that each carrier is affected by the full external magnetic field as its orbit expands, rather than one that is partially compensated by the magnetic field created by the expanding orbits of other carriers, in order that its azimuthal speed reaches the final value Eq. (51). Note in particular that within the enlarged orbits at the phase boundary in Fig. 8 (left panel) there are small normal orbits that will expand next, in a net magnetic field that is already weaker because of the field generated by the larger orbits enclosing them. However these smaller expanding orbits should not be affected by the magnetic field (in direction opposite to the applied field) created by the already enlarged orbits, because these carriers are all becoming part of the same $\Psi(\vec{r})$, hence they do not affect each other.

To clarify this point further, we show in Fig. 9 the process of expansion of a single orbit. As the orbit cuts through the magnetic field lines the electron acquires azimuthal velocity $v_{\theta}$ due to the Lorentz force, which reaches the value Eq. (51) when the orbit reaches radius 


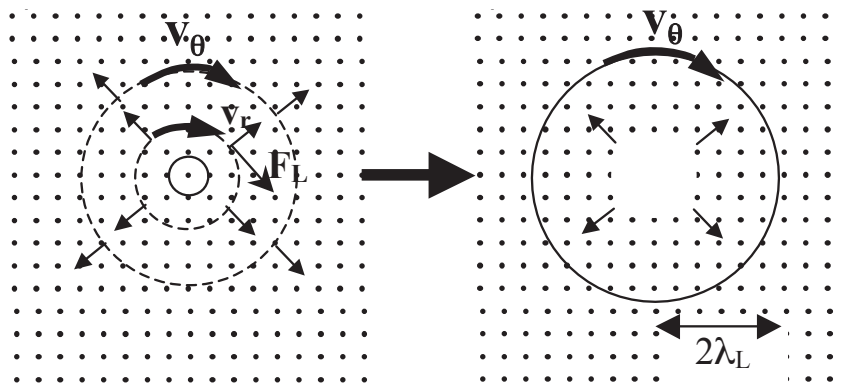

FIG. 9: A single orbit expanding in the magnetic field. As the orbit cuts through the field lines (left panel) it acquires azimuthal velocity $v_{\theta}$ reaching the value Eq. (27) when the radius reaches $2 \lambda_{L}$. When the magnetic field lines move out ( right panel) they do not affect the azimuthal velocity because different parts of $\Psi(\vec{r})$ do not affect each other.

$2 \lambda_{L}$. Then, the magnetic field lines move out cutting through the $2 \lambda_{L}$ orbit because of the compensating magnetic field generated by all the carriers in $\Psi(\vec{r})$. This would slow down the azimuthal motion due to Faraday's law if this was a normal carrier. Instead, because the carrier is part of $\Psi(\vec{r})$ its azimuthal speed is not affected as shown in the right panel of Fig. 9. In reality, the processes shown on the left and right panels of Fig. 9 do not occur sequentially but simultaneously.

We conclude from this analysis that in the transition to superconductivity, in addition to the Cooper pairing predicted by the conventional theory, the individual carriers forming the Cooper pair expand their orbits from microscopic dimension $\left(k_{F}^{-1}\right)$ to radius $2 \lambda_{L}$, without being affected by the magnetic field generated by other carriers becoming part of the same macroscopic wavefunction, and that this physics resolves the puzzle of the Meissner effect.

In the right panel of Fig. 8 we show schematically the charge distribution. Because the expansion of the orbits involves some outward charge motion, our theory predicts that associated with the $2 \lambda_{L}$ orbits there is an excess negative charge near the boundary of the superconducting region that spills over into the normal region $[45,46]$. Thus the outward motion of the phase boundary can be understood both as associated with outward motion of negative charge into the normal region and associated with enlargement of orbits from radius $k_{F}^{-1}$ to radius $2 \lambda_{L}$. The driving force for expansion of the orbits and associated negative charge expulsion is lowering of quantum kinetic energy, which is what drives superconductivity according to the theory of hole superconductivity $[47,48]$.

\section{X. $2 \lambda_{L}$ ORBITS AND THE LONDON MOMENT}

The London moment is the magnetic moment generated by a rotating superconductor [35]. The magnetic field that exists throughout the interior of a supercon- ductor rotating with angular velocity $\vec{\omega}_{0}$ is

$$
\vec{B}=-\frac{2 m_{e} c}{e} \vec{\omega}_{0}
$$

The resulting magnetization for a cylinder rotating around its axis is

$$
M=\frac{B}{4 \pi}=-\frac{m_{e} c}{2 \pi e} \omega_{0}
$$

Assuming as in Eq. (32) that each supercarrier contributes magnetic moment $\vec{\mu}$ to the magnetization yields

$$
\mu=\frac{m_{e} c}{2 \pi e n_{s}} \omega_{0}=\frac{2 e}{c} \lambda_{L}^{2} \omega_{0}
$$

and the relation Eq. (34) between magnetic moment and angular momentum yields

$$
\ell=m_{e}\left(2 \lambda_{L} \omega_{0}\right)\left(2 \lambda_{L}\right)
$$

which describes carriers in orbits of radius $2 \lambda_{L}$ and tangential velocity $2 \lambda_{L} \omega_{0}$. This provides additional support to the interpretation that carriers in the superconducting state reside in orbits of radius $2 \lambda_{L}$. The fact that the London moment is parallel rather than antiparallel to the angular velocity demonstrates that the carriers forming the superfluid have negative charge [49].

\section{SPIN MEISSNER EFFECT}

The Spin Meissner effect [39] is the spontaneous generation of a spin current within a London penetration depth of the surface of a superconductor when a metal is cooled into the superconducting state, given by

$$
\vec{J}_{\sigma}=n_{s} \vec{v}_{\sigma}^{0}=-n_{s} \frac{\hbar}{4 m_{e} \lambda_{L}} \vec{\sigma} \times \hat{n}
$$

where $\hat{n}$ is the outward-pointing normal to the surface of the superconductor and $\vec{\sigma}$ is parallel to the surface. The magnitude of the mass transport associated with this current in each direction is half the mass transport of the critical current of the superconductor. When a magnetic field $\vec{B}$ is applied the spin current component with $\vec{\sigma}$ parallel to $\vec{B}$ slows down and the one with opposite spin direction speeds up, and when the magnetic field is such that the slower spin current component is stopped the superconducting state is destroyed [39].

The negative charge expulsion predicted by our theory [45] has as consequence that an outward-pointing electric field exists in the interior of superconductors at sufficiently low temperatures. Because of this, a spin current originating in the Rashba spin-orbit interaction is expected. However, the magnitude of the spin current given by Eq. (56) is orders of magnitude larger than would be expected from the ordinary Rashba effect $[46,50]$. 
The spin-orbit interaction of an electron in the presence of an electric field $\vec{E}$ obtained from the Dirac Hamiltonian is

$$
H_{\text {s.o. }}=-\frac{e \hbar}{4 m_{e}^{2} c^{2}} \vec{\sigma} \cdot(\vec{E} \times \vec{p}) .
$$

This can be represented by the Aharonov-Casher vector potential $[51,52] \vec{A}_{\sigma}$ in the single-particle Hamiltonian

$$
\begin{gathered}
H=\frac{1}{2 m_{e}}\left(\vec{p}-\frac{e}{c} \vec{A}_{\sigma}\right)^{2} \\
\vec{A}_{\sigma}=\frac{\hbar}{4 m_{e} c} \vec{\sigma} \times \vec{E} .
\end{gathered}
$$

The term linear in $\overrightarrow{A_{\sigma}}$ from Eq. (58a) yields Eq. (57) (for an interpretation of the term quadratic in $A_{\sigma}$ see ref. [53]). Just like the ordinary vector potential $\vec{A}$ gives rise to a magnetic field $\vec{B}=\vec{\nabla} \times \vec{A}$, the spin-orbit vector potential $\vec{A}_{\sigma}$ gives rise to an effective magnetic field [39]

$$
\vec{B}_{\sigma}=\vec{\nabla} \times \overrightarrow{A_{\sigma}}=\frac{\hbar}{4 m_{e} c}(\vec{\nabla} \cdot \vec{E}) \vec{\sigma}=\frac{\pi \hbar}{m_{e} c} \rho \vec{\sigma}
$$

that imparts an azimuthal velocity to the carriers in the expanding orbits, just as the ordinary magnetic field does[44]. Here, $\rho=\vec{\nabla} \cdot \vec{E} / 4 \pi$ is the charge density that gives rise to the electric field $\vec{E}$ with which the magnetic moment of the electron interacts.

The question now is, what is this charge density $\rho$ ? The superfluid density $n_{s}$ has associated with it a charge density $q n_{s}$, with $q$ the charge of an individual carrier. Experiments such as the London moment, the gyromagnetic effect and the Bernoulli effect indicate that the charge carriers in the superfluid are electrons, hence $q=e$ [49]. Again we have to assume that the superconducting fluid described by $\Psi(\vec{r})$ does not screen itself and as a consequence the spin-orbit interaction affecting each superfluid carrier results from the electric field generated by the full compensating ionic charge density

$$
\rho=|e| n_{s}
$$

rather than from the slight net charge imbalance resulting from charge expulsion, which is smaller than Eq. (60) by a factor $v_{\sigma}^{0} / c$ [46]. Replacing Eq. (60) in Eq. (59) and using Eq. (30) for $\lambda_{L}$ yields

$$
\vec{B}_{\sigma}=\frac{\pi \hbar}{m_{e} c}|e| n_{s} \vec{\sigma}=-\frac{\hbar c}{4 e \lambda_{L}^{2}} \vec{\sigma} .
$$

When the orbit expands to radius $2 \lambda_{L}$, the azimuthal speed acquired is

$$
v_{\sigma}^{0}=-\frac{e \lambda_{L}}{m_{e} c} B_{\sigma}=\frac{\hbar}{4 m_{e} \lambda_{L}} .
$$

and just like for the Meissner effect, the internal motions cancel out and a spin current remains within $\lambda_{L}$ of the surface, given by Eq. (56). The direction of the spin current is as given in Eq. (56). The angular momentum of electrons in $2 \lambda_{L}$ orbits with speed given by Eq. (62) is

$$
\ell=m_{e} v_{\sigma}^{0} \times\left(2 \lambda_{L}\right)=\frac{\hbar}{2}
$$

the same as the intrisic electron angular momentum due to spin.

The condition Eq. (63) presumably has a topological origin and is what determines that the orbits expand to radius $2 \lambda_{L}$, which coincidentally is precisely what is needed to generate a magnetic field of just the right magnitude to cancel the external magnetic field and give rise to the Meissner effect. The driving force for the orbit expansion is lowering of quantum kinetic energy in the transition to the superconducting state [47, 53]. We regard the fact that the result Eq. (63) results from this analysis to be compelling evidence in favor of the validity of this model for the description of real superconductors.

\section{MACROSCOPIC PHASE COHERENCE}

Macroscopic phase coherence is a hallmark of superconductivity [2, 23, 27, 28]. The BCS wavefunction exhibits macroscopic phase coherence, however it does not provide an intuitive picture of what macroscopic phase coherence means, nor how it is established in the transition from the normal to the superconducting state, nor how it is robustly maintained over macroscopic distances in the superconducting state, nor how the establishment of phase coherence is related to the Meissner effect.

Instead, the theory discussed here provides a unified explanation for how superconductors expel magnetic fields and how macroscopic phase coherence is established. Within our theory the superfluid wavefunction is composed of paired orbits of spin up and spin down electrons, each orbit of radius $2 \lambda_{L}$, with distance between the centers of the orbits $\xi$, the superconducting coherence length [39]. We can think of the "phase" as a point in the electron's orbit that is rotating with angular velocity $\omega=v_{\sigma}^{0} /\left(2 \lambda_{L}\right)=|e| /\left(2 m_{e} c\right) B_{\sigma}$. As the carriers condense into the superconducting state their orbits expand and overlap with each other, and this gives rise to phase coherence because overlapping orbits have to have the same phase to avoid collisions, as shown schematically in Fig. 10. We can easily understand that this phase coherence has to extend over the entire region occupied by the superfluid wavefunction $\Psi(\vec{r})$.

\section{SUMMARY AND DISCUSSION}

In this paper we have argued that the generally held view that the conventional theory of superconductivity describes, explains and predicts the Meissner effect is incorrect. The Meissner effect is the process by which 


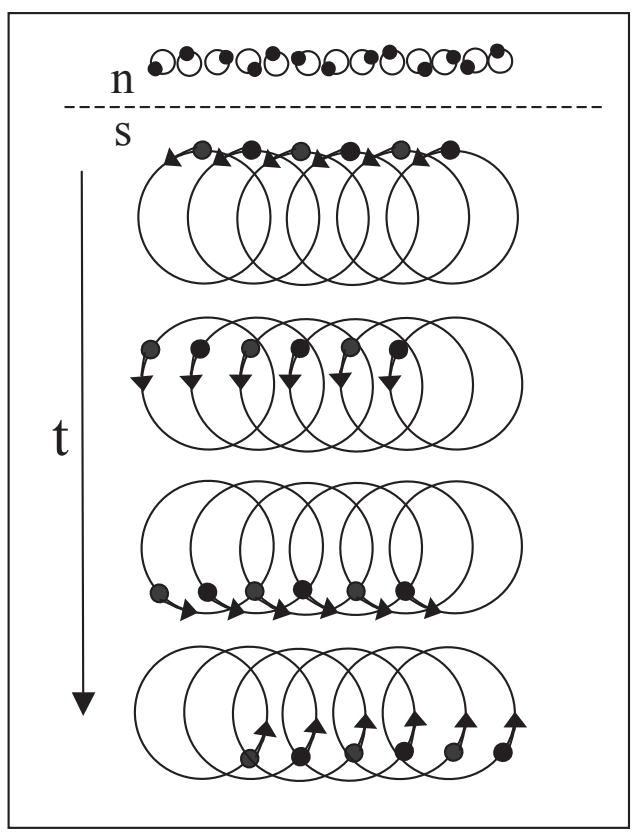

FIG. 10: The "phase" is depicted as a black circle on the orbit. In the normal (n) phase (small orbits of radius $k_{F}^{-1}$ ) there is no correlation between the phases of different orbits because they are non-overlapping. In the superconducting (s) phase (large orbits of radius $2 \lambda_{L}$ ) the orbits overlap strongly and the phases have to be the same in the different orbits to avoid collisions. As time (t) progresses the phases rotate together with angular velocity $\omega=(2 \pi / \hbar) n_{s} \mu_{B}^{2}$. The overlap between the orbits enforces the long-range phase coherence.

a metal becoming superconducting expels the magnetic field from its interior. It is generally a very non-trivial question whether a many-body system will reach its lowest energy state predicted by thermodynamics. For example a ferromagnet cooled below its critical temperature will not in general achieve an ordered state with macroscopic magnetization but rather break up into domains. A liquid when cooled will often end up as a solid glass or a polycrystal rather than a macroscopically ordered single crystal. In these processes the forces at play are well studied and understood. In contrast it is remarkable that it is generally assumed that this question is not relevant to superconductors. How superconductors achieve the state with the magnetic field expelled is not regarded to be an open question in the field [1]. The question has not even been posed, let alone been answered, in the vast literature dealing with the conventional theory of superconductivity. We suggest that the question has not been posed nor answered because the conventional theory lacks essential physical ingredients that are necessary to answer it.

It has been argued that the explanation (or lack thereof) of the Meissner effect is in essence the same as that of flux quantization in superconducting rings $[54,55]$. We argue that this is not quite so, even though the phenomena are certainly closely related. Flux quanti- zation involves changes in a fraction of the flux quantum

$$
\phi_{0}=\frac{h c}{2 e}=\frac{\pi}{\alpha} e
$$

with $\alpha=e^{2} /(\hbar c) \sim 1 / 137$ the fine structure constant. Eq. (64) is a microscopic quantity $(\sim 430$ electron charges). How superconducting rings manage to adjust their current flow in order to respect flux quantization is certainly a fundamental question, as discussed extensively by A.V. Nikulov [54], for which we don't have a dynamical explanation. However, expulsion of a 200 Gauss magnetic field from a sample of cross section $1 \mathrm{~cm}^{2}$ is a much bigger question: it involves getting rid of some $10^{9}$ flux quanta! This is a macroscopic phenomenon for which it is reasonable to expect an explanation that is consistent with the macroscopic laws of physics. The fact that we don't have a dynamical explanation of flux quantization is not in our view a valid reason to argue that a dynamical explanation of the Meissner effect is not required from the 60-year old theory that claims to explain conventional superconductivity [55].

It is very suggestive that the process of expulsion of magnetic field and transition to the superconducting state of a macroscopic sample occurs through radial expansion of small superconducting regions (Fig. 1(b) or 1(c)), rather than uniformly as depicted in Fig. 1(a). We argue that this known experimental fact gives a vivid image of the underlying physics, and have provided here two complementarly closely related physical explanations: (i) expansion of a perfectly conducting fluid (Fig. 2(b)) leads to expulsion of magnetic field, as is well known in plasma physics (Alfven's theorem [56, 57]), and (ii) expansion of electronic orbits leads to increased Larmor diamagnetism (Eq. (40)), as is well known in atomic and solid state physics [18]. In both processes the dynamical explanation for the magnetic field expulsion is the magnetic Lorentz force [58] acting on radially outgoing charge. The driving force for a radial expansion is naturally a pressure, in our interpretation quantum pressure driven by reduction of quantum kinetic energy [38, 47, 59]. The postulated outflow of superconducting carriers and backflow of normal carriers (Fig. 4) strongly resembles processes known to exist in ${ }^{4} \mathrm{He}$, where superfluid thrusts from colder to warmer regions and normal fluid backflows from warmer to colder regions (fountain effect) [60]. In superfluid ${ }^{4} \mathrm{He}$ the driving force for the transition is known to be lowering of quantum kinetic energy [59]. Finally, within our model the radial expansion of the phase boundary also gives a macroscopic image of the microscopic atomic orbital expansion that gives rise to superconductivity as described by the dynamic Hubbard model [48].

In contrast, within conventional BCS theory there is no radial charge flow associated with the radial expansion of the phase boundary, hence the driving force for the azimuthal current cannot be the magnetic Lorentz force and remains unidentified. The driving force for the radial expansion of the phase boundary is termed 'Meiss- 
ner pressure' by F. London [35] but is not given a physical interpretation, and as a consequence the expansion of the phase boundary bears no relationship to the underlying microscopic physics believed to be responsible for superconductivity within BCS [6], namely the Fröhlich electron-phonon interaction and Cooper pairing. It could be said that conventional BCS theory describes the 'expansion' of phase coherence as the superconducting nucleus expands. Then, BCS theorists have to explain how the expansion of phase coherence causes an azimuthal force to act on charge carriers. Perhaps this is a new force of nature that has not yet been identified [61] and will make BCS become part of the 'reductionist frontier' [62]. In our model instead, no new forces are needed and the development of phase coherence is explained by the expansion of the orbits to become strongly overlapping (Fig. 10), which in turn is directly linked to the azimuthal force acting on charge carriers (Fig. 9).

We argue that since the conventional theory does not describe charge expulsion it cannot describe the Meissner effect. More generally, a theory of superconductivity that can explain the Meissner effect by expulsion of charge has to know the difference between positive and negative charge, just as superconductors do [49]. We argue that this rules out all theories of superconductivity that are electron-hole symmetric, as most theories including the conventional theory are. Furthermore, since the expulsion of charge carries along an increase in potential (Coulomb) energy, we argue that this rules out any theory of superconductivity where the condensation energy is potential rather than kinetic which is the case of most theories including the conventional theory. Within our theory, electron-hole asymmetry and kinetic energy lowering are inextricably linked [48].

We have shown that the magnetization that the superconductor develops to cancel the applied magnetic field originates in the orbital magnetic moments of electrons residing in mesoscopic orbits of radius $2 \lambda_{L}$. The charge expulsion discussed in the previous paragraphs originates in the orbit enlargement from microscopic radius $k_{F}^{-1}$ to radius $2 \lambda_{L}$ in the transition to superconductivity [46]. In the absence of an applied magnetic field, electrons in these orbits give rise to a Spin Meissner effect [39], the existence of a macroscopic spin current within a Lon- don penetration depth of the surface of superconductors. The dynamical generation of the spin current and the expulsion of external magnetic fields occur through the same process, the expansion of the electron orbit from microscopic radius to the mesoscopic radius $2 \lambda_{L}$ in the presence of external magnetic field and internal electric field from the background ionic charge distribution. The fact that the speed of electrons in these orbits gives rise to angular momentum of value precisely $\hbar / 2$ [39] we regard as compelling evidence that the theory applies to nature.

Finally we have pointed out that to understand both the Meissner and the Spin Meissner effects it is necessary to assume that different parts of the macroscopic superfluid wavefunction $\Psi(\vec{r})$ do not influence each other [43], i.e. the magnetic field generated by electrons in overlapping orbits does not affect the magnetic field sensed by a given electron, and the ionic background electric field giving rise to the spin-Meissner current is not screened by the charge of electrons in overlapping orbits. This is consistent with the fact that the macroscopic wavefunction of the superconductor $\Psi(\vec{r})$ is in many ways similar to the wavefunction $\Psi(\vec{r})$ of a single electron [26].

The process of negative charge expulsion and existence of mesoscopic orbits described here gives rise to a small charge inhomogeneity over the entire macroscopic sample with the region within $\lambda_{L}$ of the surface having a small excess negative charge. The resulting macroscopic equilibrium electrodynamics equations giving the spatial distribution and quantitative values of the charge density, electric field and spin current in the ground state of superconductors are given in other publications [45, 46]. A valid microscopic theory of superconductivity will have to be consistent with these macroscopic and mesoscopic properties.

\section{Acknowledgments}

The author is grateful to D.J. Scalapino, A. J. Leggett and N. Goldenfeld for discussions on the conventional understanding of the Meissner effect.
[1] "BCS: 50 years", ed. by L. N Cooper and D. Feldman, World Scientific, Singapore, 2011.

[2] M. Tinkham, "Introduction to superconductivity", McGraw Hill, New York, 1996.

[3] W.A.B. Evans and G. Rickayzen, 'On the equivalence of the phenomena of the Meissner Effect and infinite conductivity in superconductors', Annals of Physics 33, 275 (1965).

[4] O. Klein and J. Linhard, Rev. Mod. Phys. 17, 305 (1945).

[5] J. Bardeen, Encyclopedia of Physics (Springer Verlag,
Berlin, 1956), Vol. 15, p. 274.

[6] J. Bardeen, L.N. Cooper and J.R. Schrieffer, 'Theory of Superconductivity', Phys. Rev. 108, 1175 (1957)

[7] P. G. de Gennes, "Supercomductivity of Metals and Alloys", Addison-Wesley, Redwood, City, CA, 1989.

[8] G. Lippmann, "Sur les proprietes des circuits electriques denues de resistance," Academie des Sciences, Comptes Rendus, 168, 73 (1919).

[9] H. K. Onnes, Comm. Phys. Lab. Leyden Suppl. 50a, 1924.

[10] W. Meissner and R. Ochsenfeld, "Ein neuer 
Effekt bei Eintritt der Supraleitfähigkeit", Naturwissenschaften 21, 787 (1933).

[11] H. Essen and M.C.N. Fiolhais, 'Meissner effect, diamagnetism, and classical physics - a review', Am. J. Phys. 80. 164 (2012); M.C.N. Fiolhais and H. Essen, 'Electrodynamics of Perfect Conductors', Int J Theor Phys 52, 1701 (2013).

[12] W. F. Edwards, 'Classical Derivation of the London Equations', Phys. Rev. Lett. 47, 1863 (1981).

[13] A. M. Gulian, 'A Purely Classical Derivation of the Meissner Effect?', arXiv:1201.6066 (2012).

[14] D. Yoshioka, 'Meissner effect cannot be explained classically', arXiv:1203.2227 (2012).

[15] S. Putterman, 'Comments on the variational principle and superfluid mechanics', Phys. Lett. A 89, 146 (1982).

[16] J. B. Taylor, 'A classical derivation of the Meissner effect?', Nature 299, 681 (1982).

[17] See references in http://physics.ucsd.edu/ jorge/hole.html.

[18] N. W. Ashcroft and N. D. Mermin, "Solid State Physics", Ch. 34, Saunders College Publishing, Fort Worth, 1976.

[19] A. A. Abrikosov, "Fundamentals of the Theory of Metals", Chpt. 15, North Holland, Amsterdam, 1988.

[20] J. R. Schrieffer, "Theory of Superconductivity", Chpt. 1, Addison Wesley, Redwood City, 1964.

[21] M. R. Schafroth, Helv. Phys. Acta 24, 645 (1951). J. Bardeen, Phys. Rev. 97, 1724 (1955); T. Matsubara, Prog. Theor. Phys. 13, 631 (1955); J. Bardeen, L. N. Cooper, and J. R. Schrieffer, Phys. Rev. 108, 1175 (1957); P. W. Anderson, $\quad$ Phys. Rev. 110, 827 (1958); G. Rickayzen, Phys. Rev. 111, 817 (1958); Phys. Rev. Lett. 2, 90 (1959); Phys. Rev. 115, 795 (1959);

Wentzel, $\quad$ Phys. Rev. 111, 1488 (1958); Phys. Rev. Lett. 2, 33 (1959); D. Pines and J. R. Schrieffer, Phys. Rev. Lett. 1, 407 (1958); R.M. May and M.R. Schafroth, Phys. Rev. 115, 1446 (1959); Y. Nambu, Phys. Rev. 117, 648 (1960); L.P. Kadanoff and P.C. Martin, Phys. Rev. 124, 670 (1961); D.A. Uhlenbrock and B. Zumino, Phys. Rev. 133, A350 (1964).

[22] D. J. Scalapino, private communication.

[23] R. P. Feynman, "Lectures on Physics", Addison-Wesley, Boston, 1977, Vol. III, Chpt. 21.

[24] V.L. Ginzburg and L.D. Landau, Zh. Eksp. Teor. Fiz. 20, 1064 (1950). English translation in: L. D. Landau, Collected papers (Oxford: Pergamon Press, 1965) p. 546.

[25] L. P. Gorkov, Sov. Phys. JETP 9, 1364 (1959).

[26] T. W. Nee, D. Rogovin and M. O. Scully, 'Superconductivity and macroscopic quantum phenomena', Ann. of Phys. 77, 79 (1973).

[27] B. D. Josephson, Phys. Lett. 1, 251 (1962).

[28] J. E. Mercereau, in "Superconductivity", ed. by R. D. Parks, Marcel Dekker, New York, 1969, Vol. 1, Chpt. 8.

[29] H. Frahm, S. Ullah and A. T. Dorsey, 'Flux dynamics and the growth of the superconducting phase', Phys. Rev. Lett. 66, 3067 (1991); A. T. Dorsey, Ann. of Phys. 233, 248 (1994).

[30] F. Liu, M. Mondello and N. Goldenfeld, 'Kinetics of the superconducting transition', Phys. Rev. Lett. 66, 3071 (1991).

[31] A. Schmid, Phys. Kondens. Mater. 5, 302 (1966).

[32] E. Abrahams and T. Tsuneto, 'Time Variation of the Ginzburg-Landau Order Parameter', Phys. Rev. 152, 416 (1966).
[33] R.L. Frank, C. Hainzl, B. Schlein and R. Seiringer, arXiv:1504.05885 (2015).

[34] C. Hainzl and J. Seyrich, arXiv:1504.05881 (2015).

[35] F. London, "Superfluids", Vol. I, Dover, New York, 1961.

[36] J. E. Hirsch, arXiv:1504.05190 (2015).

[37] T.E. Faber, 'Creation and Growth of Superconducting Nuclei', Nature 164, 277 (1949).

[38] J.E. Hirsch, 'Electromotive Forces and the Meissner Effect Puzzle', J. Sup. Nov. Mag. 23, 309 (2010).

[39] J.E. Hirsch, Europhys. Lett. 81, 67003 (2008).

[40] A. B. Pippard, IEEE Transactions on Magnetics 23, 371 (1987).

[41] M. Tinkham, 'The electromagnetic properties of superconductors', Rev. Mod. Phys. 46, 587 (1974).

[42] J. E. Hirsch, 'The missing angular momentum of superconductors', J. Phys. Cond. Matt. 20, 235233 (2008).

[43] E. Schrödinger, "The meaning of wave mechanics", July colloquium, Dublin 1952, in "The Interpretation of Quantum Mechanics: Dublin Seminars (1949-1955) and Other Unpublished Essays", by E. Schrödinger, Ox Bow Press, Woodbridge, CT, 1995.

[44] J.E. Hirsch, J. Supercond. Nov. Magn. 22, 131 (2009).

[45] J.E. Hirsch, 'Charge expulsion and electric field in superconductors', Phys.Rev. B 68, 184502 (2003); Phys.Rev. B 69, 214515 (2004).

[46] J.E. Hirsch, 'Electrodynamics of spin currents in superconductors', Ann. Phys. (Berlin) 17, 380 (2008).

[47] J. E. Hirsch, 'Kinetic energy driven superconductivity, the origin of the Meissner effect, and the reductionist frontier', Int. J. Mod. Phys. B 225, 1173 (2011).

[48] J.E. Hirsch, 'Dynamic Hubbard model: kinetic energy driven charge expulsion, charge inhomogeneity, hole superconductivity and Meissner effect', Physica Scripta 88, 035704 (2013).

[49] J.E. Hirsch, 'Electron-hole asymmetry and superconductivity', Phys, Rev, B 68, 012510 (2003); 'The London moment: what a rotating superconductor reveals about superconductivity', Physica Scripta 89, 015806 (2014).

[50] J. E. Hirsch, Physica C 470, 635 (2010).

[51] Y. Aharonov and A. Casher, Phys. Rev. Lett. 53, 519 (1984).

[52] S. Oh, C. M. Ryu, and S. S. Salk, Phys. Rev. A 50, 5320 (1994).

[53] J. E. Hirsch, J. Supercond. Nov. Magn. 26, 2239 (2013).

[54] A.V. Nikulov, 'The Meissner effect puzzle and the quantum force in superconductor', Phys.Lett. A 376, 3392 (2012); 'Quantum force in a superconductor', Phys. Rev. B 64 (2001) 012505.

[55] A.J. Leggett, private communication (2015).

[56] W.A. Newcomb, 'Motion of magnetic lines of force', Ann. Phys. 3, 347 (1958).

[57] D.P. Stern, 'The motion of magnetic field lines', Space Science Reviews 6, 147 (1966).

[58] J. E. Hirsch, 'The Lorentz force and superconductivity', Phys. Lett. A 315, 474 (2003).

[59] J. E. Hirsch, 'Kinetic energy driven superfluidity and superconductivity and the origin of the Meissner effect', Physica C 493, 18 (2013).

[60] F. London, "Superfluids", Vol. II, Dover, New York, 1964.

[61] S. Weinberg, 'The Forces of Nature', American Scientist 65, 171 (1977).

[62] S. Weinberg, 'From BCS to the LHC', Int. J. Mod. Phys. A 23, 1627 (2008). 